\title{
La educación superior ante la pandemia de la COVID-19: el caso de México
}

\author{
SylVia SCHMELKES
}

Socióloga con Maestría en Investigación Educativa por la Universidad Iberoamericana Ciudad de México. Vicerrectora Académica de la Universidad Iberoamericana Ciudad de México.

\section{Resumen}

El propósito de este artículo es describir los probables impactos a corto y largo plazo de la pandemia en la educación superior. Situaremos el contexto de la educación superior en México comentando la ausencia las políticas públicas educativas frente a la pandemia en el caso de este nivel educativo. Analizaremos tres factores que confluyen en el impacto sobre la educación superior: el económico, el tecnológico y el organizativo-pedagógico. Describiremos los tres grandes impactos a consecuencia de la confluencia de estos factores: la ampliación de la desigualdad educativa, el abandono escolar y el déficit de aprendizajes debido a las deficientes condiciones en los hogares. Señala la necesidad de la planeación tanto gubernamental como desde las instituciones y subsistemas para mitigar y para intentar revertir los efectos analizados. 


\title{
O Ensino Superior perante a pandemia do Covid-19: O caso do México
}

\section{Sumario}

O propósito do artigo é descrever os prováveis impactos a curto e longo prazo da pandemia no Ensino Superior. Vamos situar o contexto do Ensino Superior no México, comentando a ausência das políticas públicas educacionais perante a pandemia, no caso de esse nível educativo. Vamos analisar três fatores que confluem no impacto sobre o Ensino Superior: o económico, o tecnológico e o organizativo-pedagógico. Vamos descrever os três grandes impactos, a consequência da confluência de esses fatores: a ampliação da desigualdade educacional, o abandono escolar e o déficit de aprendizagens devido às deficientes condições nas casas. Se fala da necessidade do planejamento, assim governamental como das instituições e subsistemas, para mitigar e para tentar reverter os efeitos analisados.

Palavras-chave: custo educacional, impacto, pandemia, desigualdade, nova lei de Educação.

\section{Higher Education in the Presence of the COVID-19 Pan- demic: The Mexico Case}

\begin{abstract}
The aim of this study is to describe the probable short and long-term impact of the COVID-19 pandemic on higher education. We situate this subject in Mexico by considering and commenting on the public education policies absence during the pandemic. We analyze the three factors that converge in the impact on higher education: economic, technological and pedagogical-organizational. Then, we describe the three major impacts as a result of the confluence of those factors: educational inequality expansion, school dropout and deficit of learning due to the poor conditions at home. The results show the need for governmental and institutional planning, and subsystems that mitigate and reverse the pandemic effects on higher education.
\end{abstract}

Keywords: Educational cost, Impact, Pandemic, Inequality, New education law. 


\section{Una rápida visión de la situación de la educación superior en México}

La población mexicana entre 24 y 65 años con un título de educación superior está apenas $17 \%$, por debajo de otros países de la región como Chile (23\%), Colombia (23\%), Costa Rica (23\%) y Argentina (21\%) (OECD, 2019a). Por estas fechas, hay alrededor de 3.9 millones de estudiantes matriculados en programas escolarizados y 760,000 en programas no escolarizados, en más de 5,000 instituciones de educación superior, de las cuales 2,180 son públicas. La inmensa mayoría (91\%) se encuentran inscritos en licenciatura (SEP, 2019). Ello representa una cobertura bruta de $40 \%$ (Silva y García, 2020), menor que la que reporta Argentina, Chile, Uruguay y Venezuela.

El sistema de educación superior es altamente complejo. Consta de 13 subsistemas muy diferentes entre sí. La matrícula se concentra en la licenciatura (89\%). Sólo 5.9\% corresponde a especialización y maestría, y 1\% a doctorado (OECD, 2019a). Los distintos subsistemas de educación superior no se encuentran vinculados entre sí y no existe, a la fecha, un cuerpo que los articule. La movilidad entre subsistemas es prácticamente imposible.

La situación de la educación superior en México arrastra problemas crónicos. Quizás el más importante de ellos, a pesar de la masificación que ha sufrido en las últimas seis décadas, es su atención a un sector privilegiado de la sociedad y la subrepresentación de los que pertenecen a los deciles inferiores. Así, sólo 8.5\% de la matrícula proviene de los dos primeros deciles, a pesar de que en la población general los jóvenes de estos deciles representan $21.1 \%$. $27.7 \%$ proviene de los últimos dos deciles, que sólo agrupan a $12.3 \%$ de la población. Además, en la educación superior hay una escasa representación de la población rural, indígena, habitante de zonas marginadas y con alguna discapacidad. La desigualdad es también geográfica. Así, si bien en la ciudad de México la tasa bruta de cobertura alcanza 95\%, en las entidades más pobres del país como Guerrero, Oaxaca y Chiapas, este porcentaje apenas supera 20\% (Silva y García, 2020). La oferta de educación superior se encuentra claramente concentrada geográficamente, y representa también, como en la generalidad de los países, una oferta ubicada mayoritariamente en las ciudades medianas y grandes. 


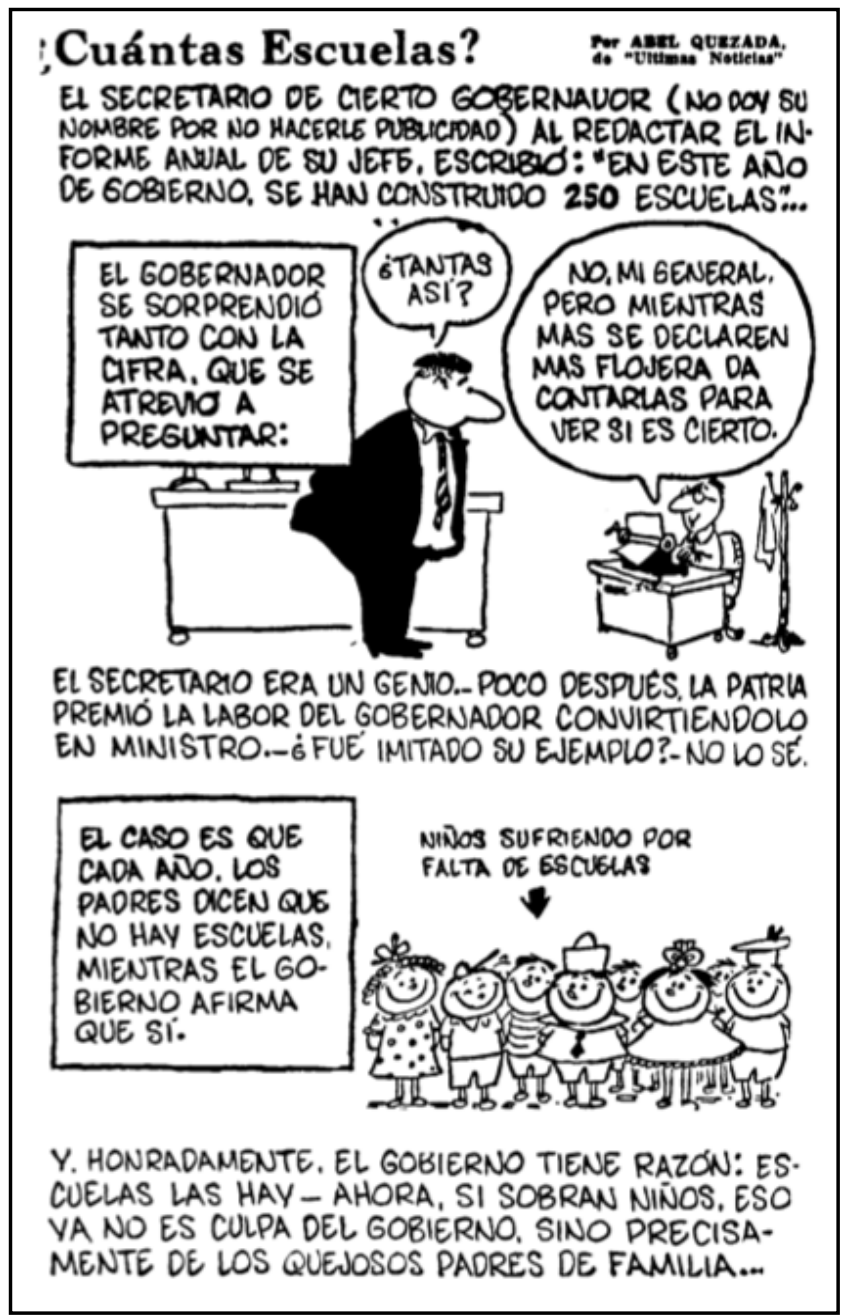

Excélsior. Número 14,469. Página 6-A. Sábado 9 de febrero, 1957.
Un problema relacionado tiene que ver con la segmentación de la calidad de la educación superior en el país. Se encuentran en un sitio privilegiado las universidades autónomas, ubicadas en su gran mayoría en las capitales de las entidades federativas. En las condiciones más precarias se encuentran la mayoría de las 264 escuelas normales (que forman docentes a nivel terciario) y las universidades interculturales. El sector privado representa una parte considerable de la matrícula (cerca de $40 \%)$ de la oferta escolarizada (Cruz López y Cruz, 2008), y alrededor de $30 \%$ de la oferta total (SEP, 2019), y su participación en la misma, ha venido aumentando en las últimas décadas como efecto del lento crecimiento de la educación superior pública. A su interior, sin embargo, el sector de educación superior privada también se encuentra fuertemente segmentado, pues existen unas cuantas universidades privadas de muy alta calidad, mientras que muchas de ellas son pequeños negocios que ofrecen carreras que requieren muy poca inversión.

La eficiencia terminal de la educación superior es sumamente baja, y el tránsito por las carreras altamente irregular. El abandono anual es de $8.9 \%$ (SEP, 2019), y los cálculos de la eficiencia terminal varían según la metodología elegida para calcularla y dependiendo si la definición es sobre el egreso o sobre la titulación, pero tomando este último, se calcula en cifras bajas, menores a $30 \%$, en los tiempos ideales. 
El costo de la educación superior en México es muy alto cuando se le compara con el de otros países de la OCDE. El costo per cápita de este nivel educativo triplica el de la educación básica, cuando en los otros países de la organización es apenas 50\% superior. A pesar de ello, el presupuesto no resulta suficiente para enfrentar los gastos, incluso los ordinarios, del funcionamiento de muchas universidades. Esto ocurre a pesar de que en las instituciones la mayor parte de los profesores son de asignatura (entre 70 y $80 \%)$.

\section{El contexto inmediato}

La pandemia llega a un año y unos cuantos meses de que Andrés Manuel López Obrador, del Partido MORENA, asume la presidencia de la República. Llegó al poder con 53\% de los votos, un gran apoyo popular. Propuso y comenzó a implementar cambios muy importantes en la política pública en general, y en particular en la educativa. A los seis meses de su toma de posesión se aprobó un cambio en el Artículo $3^{\circ}$ Constitucional que marcó una clara ruptura con la política educativa de la anterior administración. La primera iniciativa que llegó al congreso había suprimido el párrafo relativo a la autonomía universitaria, supuestamente por error. Las protestas inmediatas condujeron a su reinserción. En lo relativo a educación superior, establece la obligatoriedad y la gratuidad de este nivel educativo, y prevé la creación de un fondo que lo vaya haciendo gradualmente posible. En materia de política de educación superior, las innovaciones notables son las Universidades Benito Juárez, ubicadas en zonas marginadas, que ofrecen una carrera y pretenden llegar a albergar a 3,000 alumnos, así como el programa Jóvenes Escribiendo el Futuro, que consiste en becas a estudiantes de escasos recursos en educación superior. A estos programas se van los recursos presupuestales de 2019, mientras que los fondos extraordinarios destinados a las instituciones de educación superior, con los que se financiaban programas y proyectos no cubiertos por el presupuesto ordinario y destinados al crecimiento y a la mejora de la calidad, disminuyen a la mitad. En el presupuesto 2020, lo destinado a las becas Jóvenes Escribiendo el Futuro aumentan 74\%, mientras que el fondo para asegurar la obligatoriedad de la educación superior, comprometido en los transitorios de la reforma al Artículo $3^{\circ}$, no se crea (Mendoza, 2020).

La pandemia conduce al cierre de las instituciones educativas entre el 23 de marzo y el 1 de abril, fecha a partir de la cual se vuelve obligatorio por disposición de la Autoridad Federal en Salud, que ante la emergencia sanitaria determina la suspensión de actividades no esenciales, entre las cuales se encuentran las educativas, y establece un sistema de semáforo (rojo, naranja, amarillo y verde) por regiones, estipulando que las actividades educativas sólo podrán retomarse de manera presencial cuando el semáforo esté en verde. Cuando escribo estas líneas, casi seis meses después, el semáforo sigue en naranja en la mayor parte del país. Estas medidas fueron retomadas por la Secretaría de Educación Pública muy tempranamente, de manera que el 16 de marzo publica los Lineamientos de Acción Covid-19 para la Educación Superior (SEP 2020a), que sugieren el cierre de actividades el 20 de marzo y el regreso a clases presenciales el 20 de abril. Si bien estos 
lineamientos pronto se hicieron obsoletos debido a que no fueron pensados para un confinamiento prolongado, en vista de que representan el único documento de políticas frente a la pandemia, selecciono algunas de sus disposiciones:

- Consolidar vías de comunicación permanentes con sus académicos, estudiantes y trabajadores para avisos oficiales y acciones inmediatas.

- Suspender actividades masivas.

- Colocar filtros para detectar personas con alguna dificultad respiratoria.

- Establecer medidas sanitarias como la disposición de jabón y gel antibacterial.

- Generar conciencia de las medias de higiene, buena alimentación y fortalecimiento del sistema inmunológico.

- Solicitar a las personas que presentan síntomas que se queden en casa.

Entre las medidas académicas y de comunicación se señalan las siguientes:

- Contar con un repositorio nacional para que las IES puedan compartir los diversos materiales generados desde sus comunidades y sirvan a otras instituciones educativas. Los materiales tendrán que estar integrados con metadatos con la finalidad de que las búsquedas se realicen de forma adecuada.

A partir del 20 de marzo se sugiere la suspensión de actividades presenciales, aunque a las universidades autónomas se les da la posibilidad de que sus órganos colegiados tomen estas decisiones.

Las actividades administrativas deben continuar, privilegiando la vía remota o bajo el esquema de guardias, que serán establecidas por cada institución educativa. Los trabajadores administrativos y operativos de la tercera edad no deberán acudir a sus lugares de trabajo. Cada instancia generará condiciones para realizar labores desde el hogar de cada trabajador o trabajadora.

A las Universidades, Tecnológicos, Politécnicas y Universidades Interculturales se les pide preparar medidas académicas (impartición de clases por medios digitales y a distancia) y de comunicación con las y los estudiantes para ajustes del calendario y programas. Se estipula hacer uso de los canales de televisión y las radiodifusoras. Las Universidades Interculturales deben elaborar, en coordinación con el Instituto Nacional de los Pueblos Indígenas (INPI), materiales de difusión en lenguas indígenas sobre las medidas preventivas ante la COVID-19.

El 26 de abril se reúnen los rectores de las Instituciones de Educación Superior que pertenecen a la Asociación Nacional de Universidades e Instituciones de Educación Superior, con el Secretario de Educación Pública y el Subsecretario de Educación Superior y emiten un acuerdo nacional frente a la emergencia (ANUIES, 2020). En el mismo las Instituciones de Educación Superior acuerdan salvaguardar la salud de las comunidades universitarias y de la sociedad en general, asegurar la continuidad educativa de sus instituciones, apoyar científica y tecnológicamente a la sociedad y, con el sector productivo, reactivar la economía. Acuerdan transitar de modelos presenciales a modelos remotos, de educación abierta y a distancia a fin de no interrumpir el servicio educativo. 
El 22 de mayo se celebró otra reunión de los rectores y directores de instituciones de educación superior de la ANUIES con la presencia del Secretario de Educación Pública, quien hace un llamado a las mismas a vincularse a través de la investigación y la docencia con la realidad nacional para generar un modelo educativo enfocado a resolver los problemas sociales y económicos del país. Indica la intención de ir logrando que todos los estudiantes tengan acceso a la conectividad. Señala la orientación del gobierno hacia la creación de universidades en zonas indígenas y de alta marginación. Demanda cambios en los contenidos de la educación superior de manera que sean útiles y pertinentes a los problemas de la realidad nacional. Y solicita una mejor educación en salud en todos los niveles educativos.

Como puede verse, en realidad las políticas que existen fueron las que dictaron las autoridades de salud. En el caso de la educación superior, se emitieron lineamientos, que más bien fueron recomendaciones, muy al principio de la pandemia, los cuales pronto fueron obsoletos. Durante el confinamiento de mayo a julio, mes en el que cerró el ciclo escolar 2019-2020, la Secretaria de Educación Pública aplicó el programa Aprende en Casa para la educación básica y media superior. Vino después el periodo de vacaciones, de seis semanas, y el 24 agosto iniciaron nuevamente las clases a distancia mediante el programa Aprende en Casa II, al que se sumó un número importante de televisoras tanto abiertas como de cable y varias radiodifusoras. Salvo las escuelas normales, que son normadas a nivel federal por la Secretaría de Educación Pública, las instituciones de educación superior operaron conforme a sus propios cuerpos directivos o propios de su subsistema.

Poco antes de que iniciara la pandemia emergió el tema de la violencia de género en las instituciones de educación superior. En varias casas de estudio las mujeres organizadas pararon las actividades en demanda de su erradicación y de sanciones a los perpetradores. La UNAM tenía todavía algunas instalaciones tomadas por las mujeres organizadas cuando llegó el momento del confinamiento (García García, 2020).

Las instituciones de educación superior se vieron en la necesidad de transitar a la educación remota. Lo hicieron en tiempos muy distintos, dependiendo de la capacidad de cada una, o de cada subsistema, de efectuar la migración tecnológica. Las diferencias fueron importantes: la educación no escolarizada 


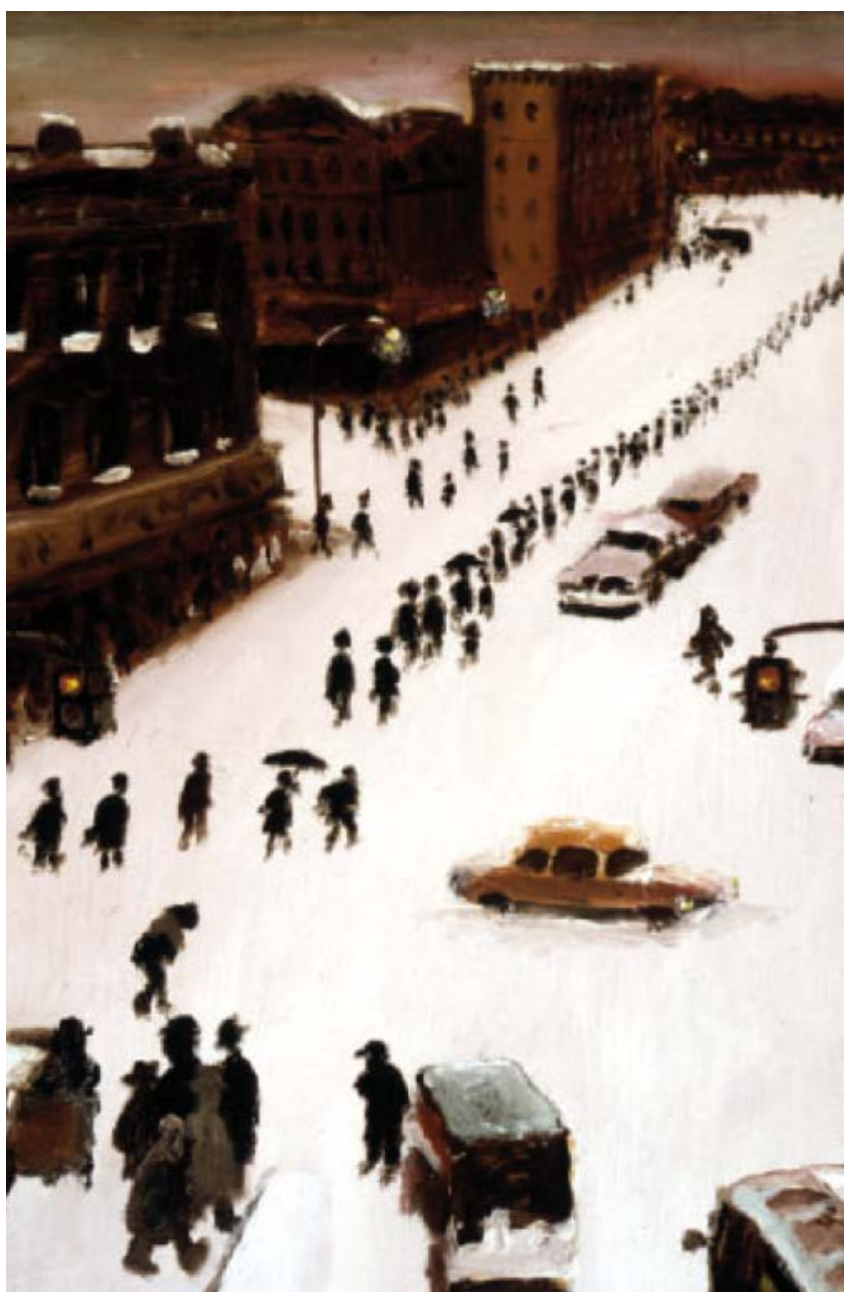

El día de la gran nevada en Jamaica, NY, en 1978 (detalle). Óleo sobre tela, 48 x $58 \mathrm{~cm} .1978$. pudo continuar sin interrupciones, al igual que algunas universidades privadas. En general las universidades $\mathrm{y}$ los institutos tecnológicos interrumpieron clases durante periodos que van entre uno y cuatro meses. Todas ellas enfrentaron, en diferente grado, la dificultad de acceso a la tecnología y al internet por parte de sus alumnos. Algunas de ellas facilitaron el acceso de los mismos a dispositivos electrónicos, mayormente tabletas o laptops. Muchas tuvieron que batallar con la falta de experiencia en el uso de la tecnología por parte de profesores y alumnos, y en todos los casos, con la falta de formación de los docentes para la educación a distancia, que también solucionó cada institución o subsistema según sus posibilidades. De parte del gobierno no hubo recursos adicionales para la educación superior pública en esta emergencia, por lo cual las instituciones tuvieron que echar mano de sus presupuestos previamente aprobados para efectuar la migración a la educación a distancia. Todas las universidades y subsistemas tuvieron que hacer ajustes en su calendario. Un ciclo escolar cerró durante la pandemia y uno nuevo se abrió. Algunas instituciones, como la Universidad Nacional Autónoma de México, el Instituto Politécnico Nacional y la Universidad Autónoma Metropolitana, por mencionar las de la ciudad de México, decidieron hacer exámenes de admisión presenciales, guardando las medidas sanitarias recomendadas. $\mathrm{La}$ 
Secretaría de Educación Pública lanzó el programa Rechazo Cero, que tiene como propósito "generar mayores oportunidades de ingreso a la educación superior, a través de la educación vocacional, laboral y la difusión de becas." Vincula a los aspirantes que participaron en el concurso de selección de alguna de las instituciones de educación superior públicas de mayor demanda y que no fueron seleccionados, con otras opciones educativas en las que participan instituciones públicas y privadas que ofrecen carreras tanto escolarizadas como de forma mixta y a distancia. Dispone para ello de cerca de 125,000 lugares (SEP, 2020b).

\section{El impacto de la pandemia en la educación superior}

Para entender la magnitud del impacto de inmediato y de largo plazo de la pandemia sobre la educación superior es necesario tener en cuenta la confluencia de tres factores: 1) la problemática económica que se deriva de la disminución de actividad productiva y comercial durante la pandemia y del consecuente desempleo o pérdida de fuentes de ingreso; 2) la enorme brecha digital que va de la mano de la desigualdad socioeconómica y se sobrepone a ella; y 3) la dificultad por parte de las instituciones de educación superior de enfrentar la crisis educativa que se deriva de la pandemia.

El primero es, desde mi punto de vista, el que más afecta la educación superior. La Encuesta Telefónica de Ocupación y Empleo (ЕTOE) reportó que:

La Tasa de Participación Económica en abril de 2020 fue de 47.5\% respecto a la población de 15 años y más, cifra inferior en 12.3 puntos porcentuales a la de marzo del mismo año. Esta disminución representa una variación a la baja de 12 millones de personas económicamente activas, principalmente por encontrarse en un estado de suspensión laboral temporal ocasionado por la cuarentena (INEGI, 2020, p.02).

Si bien ha habido una ligera recuperación durante los meses de julio y agosto gracias al inicio de la reactivación económica, todavía el impacto es muy alto sobre las familias, sobre todo de aquellas en las que algún miembro tenía un empleo formal y lo perdió, y de las que dependen de la economía informal que vio disminuida radicalmente su actividad. La Encuesta COVID-19 llevada a cabo por la Universidad Iberoamericana estima que uno de cada tres hogares observó una reducción de $50 \%$ o más de su ingreso entre febrero y marzo de 2020 (IBERO, 2020). Una encuesta llevada a cabo por Vieyra, et al., (2020) a 4800 estudiantes universitarios en México, Colombia y Perú contiene datos aún más alarmantes: 91\% de ellos han sufrido una disminución de sus ingresos, y $64 \%$ de entre ellos, una mayor a $60 \%$. Ello afecta a los jóvenes que estudian en el nivel superior de forma especial, pues se encuentran en edad de trabajar, están capacitados para hacerlo, y se ven en la necesidad de buscar trabajo y fuentes de ingreso para satisfacer las necesidades básicas de la familia. El desempleo, como sabemos, afecta proporcionalmente más a los más pobres.

El segundo es también de enorme importancia, pues el acceso a la tecnología y al internet se convirtió en la única forma de asegurar la continuidad 
de los estudios superiores. Sin embargo, la OCDE (OECD 2019b) encuentra en 2018 que en México 53\% de los jóvenes de 15 años que asisten a la escuela cuentan con computadora, y que sólo $68 \%$ tiene acceso a internet. Lo más grave es la diferencia que existe en el acceso a las computadoras en función del nivel de ingreso de la familia: sólo $11 \%$ de quienes se encuentran en el primer cuartil tiene acceso, mientras que $78 \%$ de quienes se encuentran en el cuarto cuartil lo tiene. En el caso de la educación superior la diferencia entre las escuelas privadas y públicas, con relación al acceso a internet, es también importante, pues $96 \%$ de los estudiantes de la primera cuenta con él, mientras esto es cierto en $72 \%$ de las escuelas públicas (Vieyra, et al., 2020). A lo anterior hay que agregar que los dispositivos de acceso a internet generalmente se comparten entre diversos miembros de la familia, y respecto de ello también hay una fuerte diferencia, pues si bien $55 \%$ de los estudiantes de instituciones privadas cuentan con su propio dispositivo, esto es cierto solamente en $20 \%$ de los estudiantes de instituciones públicas (Vieyra, et al., 2020).

El tercer factor es de naturaleza pedagógica. Tiene que ver con el uso que se hace de la tecnología en el entendido de que en estos tiempos es el único vehículo de enseñanza. Existe una pedagogía propia de la educación a distancia y de la educación en línea que es en gran parte desconocida por los profesores universitarios. Estos tienden a reproducir por las vías tecnológicas las clases magistrales que suelen dar en las instituciones de educación superior, pero con el agravante de que, sin la relación cara a cara con los estudiantes, el protagonismo de estos últimos se ve mermado. Además, las encuestas que se han elaborado han mostrado que los alumnos tienen serias dificultades para mantener la atención en las clases a distancia. Por otra parte, también aducen una sobrecarga de trabajos que dejan los profesores como forma de compensar la falta de presencia física (Torres, 2020; García García, 2020), lo que les deja poco tiempo para realizar otras actividades económicas, familiares o incluso de esparcimiento ${ }^{1}$.

Estos tres factores se conjugan para propiciar tres graves impactos inmediatos y mediatos sobre la educación superior en nuestro país.

El primer impacto, posiblemente el más grave, es el de la ampliación de la brecha preexistente en el acceso a la educación superior que describimos en la primera parte de este artículo. Ahora, quienes pueden tener acceso a la educación superior son quienes cuentan con computadora y acceso a internet, que como hemos visto, son sólo un porcentaje de los estudiantes universitarios y fundamentalmente los que pertenecen a grupos de ingreso más altos. Esto se sobrepone a una realidad de antemano sumamente desigual respecto del acceso y la permanencia en la educación superior que afecta también a los más pobres. Con ello, la brecha entre sectores socioeconómicos se amplía, con las graves consecuencias que ello tiene para un país democrático en el que la educación es la vía legítima privilegiada para propiciar la permeabilidad social. Ocurre en el país que ocupa el lugar 122 de 164 del Coeficiente Gini de concentración de ingreso en el mundo (BBVA, 2019). No es necesario reparar en las consecuencias de una sociedad crecientemente desigual sobre la economía (escaso poder de compra de un sector importante de la población), la política (diferencial acceso a los procedimientos democráticos previstos para transformar el statu quo, 
inestabilidad), y social (pobreza, riesgo aumentado de violencia social), así como la tragedia de un problema humano que ocurre al existir obstáculos para el crecimiento y el desarrollo de personas y comunidades.

El segundo impacto es correlativo al anterior, pero se refiere concretamente a la pérdida de estudiantes en este nivel educativo debido al abandono que es consecuencia de los tres factores ya señalados más arriba: el económico, el tecnológico y el pedagógico. Como veíamos al principio, México tiene una tasa de cobertura bruta en educación superior que lo coloca por debajo de otras economías incluso menores en la región. El porcentaje de personas mayores de 24 años con estudios universitarios es en consecuencia también inferior al de países hermanos. Sabemos que la formación de cuadros altamente capacitados es esencial para el desarrollo científico, económico y sociopolítico de las naciones. La pérdida de estudiantes estimada por la Secretaría de Educación Pública como consecuencia de la pandemia es de 8\%, lo que significa 320,000 estudiantes (Animal Político, 2020). Sin embargo, las estimaciones del PNUD duplican esta cantidad, ubicándola en cerca de 630,0000 (Factor Capital Humano, 2020). La UNAM, considerada la mejor universidad del país, calcula que $20 \%$ de sus estudiantes está en riesgo de abandono, lo que representa 72,000 alumnos en educación media superior y superior (LatinUs, 2020).

El tercer impacto es sobre la calidad de la educación superior que están recibiendo los estudiantes que han podido seguir sus estudios a distancia durante algunos de los seis meses de confinamiento que hemos tenido al escribir estas líneas. La situación en general plantea condiciones de aprendizaje que no son favorables. Muchos estudiantes no cuentan con el espacio adecuado para recibir las clases a distancia porque comparten el hogar con otros miembros, algunos de los cuales también deben conectarse para estudiar o trabajar. En algunos hogares hay enfermos de COVID, y los estudiantes pueden haber experimentado decesos de personas cercanas. El propio confinamiento afecta anímicamente a los estudiantes que no pueden vivir su vida completa y que extrañan a sus compañeros en una edad en la que éstos son esenciales. De manera especial les afecta la incertidumbre, pues justamente en la edad en la que están haciendo su plan de vida, no pueden prever lo que ocurrirá con su educación y mucho menos con su potencial trabajo o fuente de ingresos (Limón, 2020). Esta última condición, sobre todo, pero aunada a las anteriores, generan una situación altamente estresante que en ocasiones desencadena eventos depresivos y que deteriora las condiciones para aprender.

Por otra parte, no se puede negar que la educación a distancia tiene grandes virtudes. Pueden traerse a la pantalla múltiples recursos, humanos y de otro tipo, para favorecer ricas experiencias de aprendizaje. Hay cosas que se pueden hacer a distancia que no se pueden lograr presencialmente, por ejemplo la posibilidad de contar con personas de otras universidades lejanas como insumos para algunas de las clases. Pero al mismo tiempo, tiene fuertes limitaciones. Una de ellas es la descontextualización y la necesaria despersonalización de la enseñanza, que le dificulta a profesor o profesora tomar en cuenta las experiencias previas de los estudiantes, sus particulares intereses y talentos, e incluso su origen cultural, lo que en la presencia permite favorecer un enriquecimiento entre todos, estudiantes 
y profesores, y sin duda conduce a hacer más relevante el aprendizaje. Otra más es la restricción, que no es necesaria, pero ocurre, del trabajo colaborativo entre estudiantes. Una tercera es la falta de convivencia informal, antes y después, e inclusive durante las clases, que en la presencialidad siempre se da como parte integral de la experiencia de aprendizaje.

Desde luego es necesario referirnos a la imposibilidad de acceder a laboratorios y talleres que en muchas carreras universitarias son indispensables para el logro de los objetivos de formación profesional. También han tenido que cancelarse las prácticas profesionales y el servicio social, que son complementos esenciales a la formación profesional. Sin duda, cuando la pandemia esté bajo control, será necesario reponer intensivamente estas experiencias de aprendizaje. Pero la simultaneidad requerida de los laboratorios y las prácticas con las clases teóricas, por ejemplo, es innegablemente una pérdida.

Para algunas mujeres la pandemia también ha significado un incremento de la de violencia doméstica. ONU Mujeres ha señalado el peligro de que en una situación de confinamiento la violencia por razones de género es un riesgo mayor, y son las mujeres y la infancia quienes se encuentran en una peor situación (Zabalgoitia, 2020).

Todo lo anterior se traduce en la necesidad de aceptar que tendremos difíciles condiciones de aprendizaje y un consecuente déficit en los aprendizajes de la generación de estudiantes que en este momento se encuentran inscritos en cualquier nivel educativo, pero también en educación superior. Son la generación "pandemia" que corre el riesgo de ser estigmatizada en el mercado de trabajo. Los estudiantes saben esto, y ello forma parte de su incertidumbre y afecta su salud emocional.

\section{El futuro que se alcanza a visualizar}

Los impactos a los que me acabo de referir necesariamente tendrían que formar parte de un esfuerzo extraordinario de planeación para cuando esté controlada la pandemia. Si bien las instituciones de educación superior se encuentran ya pensando en ello y planeando para ello, desde el punto de vista de la acción gubernamental existen serias preocupaciones. En junio de este año, tres meses después de iniciada la pandemia, se presentó con seis meses de retraso el Programa Sectorial de Educación correspondiente a esta administración, 2020-2024. Dicho programa no hace referencia alguna a la pandemia ni a la necesidad de enfrentar sus consecuencias educativas y de remediar los daños para ningún nivel educativo. Por otra parte, recientemente se presentó el proyecto de presupuesto de egresos para 2021 que deberá ser revisado y aprobado por la Cámara de Diputados en fechas recientes. En dicho presupuesto no existe previsión alguna para atender las consecuencias educativas de la pandemia. Y si bien el presupuesto total para educación aumenta un poco, en $3 \%$, y el porcentaje de incremento para la educación superior es también de $3 \%$, hay un aumento muy grande destinado a becas universales para estudiantes de educación básica y media superior, y selectiva para estudiantes de educación superior, a costa de la cancelación de una buena cantidad de programas que estaban en marcha, 
muchos de ellos orientados a favorecer la equidad en educación básica (SHCP, 2020).

$\mathrm{Al}$ parecer se deja la respuesta a la pandemia y a sus consecuencias en manos de las instituciones y los subsistemas de educación superior, sin que para ello existan, hasta el momento, programas o políticas orientados a favorecer que se contrarresten los impactos que podemos considerar como devastadores a los que nos hemos referido. Las instituciones y los subsistemas podrán hacerlo respecto a sus propias poblaciones, y ello implica atender la desigualdad al interior de las mismas, así como recuperar el abandono y reponer el déficit de aprendizajes. Sin embargo, la desigualdad educativa a nivel sistémico, que como hemos visto se ha ahondado con la pandemia y augura futuros sociales indeseables, no tiene todavía una clara perspectiva de mitigación, pues ella sí requiere de políticas públicas decididas a combatirla.

Al cerrar estas líneas se está discutiendo en la Comisión de Educación del Senado de la República la propuesta para una nueva Ley General de Educación Superior, que vendría a sustituir a la actualmente vigente que se aprobó hace ya 42 años, en 1978 . Esta propuesta parte del espíritu del Artículo $3^{\circ}$ Constitucional recién modificado en 2019 e incluye aspectos clave como la equidad y la inclusión, la diversidad y la interculturalidad, la creación de un sistema nacional de educación superior y las formas de operacionalizar la obligatoriedad y la gratuidad de este nivel educativo estipulada en el propio artículo constitucional. Estamos a la expectativa de que la redacción final de este instrumento legal otorgue las bases necesarias para que se puedan ir atendiendo tanto los problemas históricos como los generados por la emergencia sanitaria de una manera ordenada y efectiva.

\section{Nota}

1. Estos datos proceden de encuestas en línea que Juan Carlos Silas Casillas y Sylvia Vázquez Rodríguez aplicaron a profesores universitarios durante la pandemia en varios países de América Latina (México, Venezuela, Perú, Argentina, Bolivia, Paraguay, Colombia, Ecuador, Chile, Costa Rica, Panamá y Uruguay) para estudiar cómo el profesorado vivió esa transformación de la docencia presencial hacia la virtualidad. 


\section{Referencias}

Animal Político. (09 de agosto 2020). SEP estima deserción de 10\% en educación básica y en $8 \%$ en superior por la COVID. Recuperado de: https://www.animalpolitico. com/2020/08/sep-desercion-educacion-covid/

ANUIES. Asociación Nacional de Universidades e Instituciones de Educación Superior (2020). Acuerdo nacional por la unidad en educación superior frente la emergencia sanitaria provocada por la COVID-19. Recuperado de: http://www. anuies.mx/media/docs/avisos/pdf/200424155500Acuerdo+Nacional+frente+al+COVID-19.pdf

BBVA. (06 de agosto 2019). México: Evolución de la Pobreza y Distribución del Ingreso. México: BBVA. Recuperado de: https://www.bbvaresearch.com/publicaciones/ mexico-evolucion-de-la-pobreza-y-distribucion-del-ingreso/

Cruz López, Y. y Cruz López, A. K. (2008). "La educación superior en México tendencias y desafíos". Avaliação: Revista da Avaliação da Educação Superior (Campinas), 13(2), Pp. 293-311. https://doi.org/10.1590/S1414-40772008000200004

Factor Capital Humano. (24 de agosto 2020). Dejarán sus carreras 630,000 mexicanos por la pandemia. PNUD. 24 de agosto. Recuperado de: https://factorcapitalhumano.com/carrera/dejaran-sus-carreras-640000-universitarios-mexicanos-por-la-pandemia-pnud/2020/08/

García García, C. (2020). "Reflexiones sobre la educación superior en México: ¿qué retos agrega la pandemia por COVID-19?” Faro Educativo, Apunte de política Núm. 17. Ciudad de México: INIDE-UIA. Recuperado de: https://faroeducativo. ibero.mx/2020/07/06/apunte-de-politica-n-17-reflexiones-sobre-la-educacion-superior-en-mexico-que-retos-agrega-la-pandemia-por-covid-19/

Gobierno de México. Secretaría de Educación Pública. (2020). Lineamientos de acción covid-19. Instituciones Públicas de Educación Superior. Recuperado de: http:// www.anuies.mx/recursos/pdf/LINEAMIENTOS_COVID-19_IES_SES_VFINAL.pdf

IBERO. Universidad Iberoamericana (29 de abril 2020). Encuesta de Seguimiento de los Efectos de la covid en el bienestar de los hogares mexicanos. México: Universidad Iberoamericana. Instituto de Investigaciones para el Desarrollo con Equidad. Recuperado de: https://ibero.mx/prensa/cae-por-covid-19-50-de-ingresoen-1-de-cada-3-hogares-encuesta

INEGI. Instituto Nacional de Estadística y Geografía (01 de junio 2020). "Resultados de la Encuesta Telefónica de Ocupación y Empleo (ETOE). Cifras Oportunas de abril de 2020". Comunicado de Prensa Núm. 264/20. Recuperado de: https:// www.inegi.org.mx/contenidos/saladeprensa/boletines/2020/enoe_ie/ETOE. pdf

LatinUs. (24 de septiembre 2020). La UNAM estima que 20\% de sus estudiantes están en riesgo de deserción por la pandemia. Recuperado de: https://latinus. us/2020/09/24/unam-estudiantes-riesgo-desercion-pandemia/

Limón, M. (2020). La Rebeldía. México: Valora Consultoría. Recuperado de: https:// valora.com.mx/author/miguel/

Mendoza, J. (2020). "Presupuesto federal de la educación superior en dos décadas y primeros impactos de la crisis sanitaria de 2020”. En: Educación y pandemia. Una visión académica. Pp. 92-102) México: IISUE-UNAM.

OECD. Organisation for Economic Co-operation and Development (2019a). Higher Education in Mexico: Labour Market Relevance and Outcomes, Higher Education, oecd Publishing, Paris. Recuperado de: https://read.oecd-ilibrary.org/education/higher-education-in-mexico_9789264309432-en\#page33

OECD. (2019b). PISA 2018 results. Recuperado de: https://www.oecd.org/pisa/ publications/pisa-2018-results.htm

Universidades $\mid$ núm. 86, octubre-diciembre 2020 | UDUAL $\mid$ DOI:https://doi.org/10.36888/udual.universidades.2020.86.407 Sylvia Schmelkes $\mid$ La educación superior ante la pandemia de la COVID-19: el caso de México 
SEP. Secretaría de Educación Pública (2019). Principales cifras del sistema educativo nacional. México: Secretaría de Educación Pública. Recuperado de: https://www.planeacion.sep.gob.mx/Doc/ estadistica_e_indicadores/principales_cifras/principales_cifras_2018_2019_bolsillo.pdf

SEP. (2020a). Lineamientos de Acción COVID-19 para las Instituciones Públicas de Educación Superior. México: Secretaría de Educación Pública. Recuperado de: http://www.anuies.mx/recursos/ pdf/LINEAMIENTOS_COVID-19_IES_SES_VFINAL.pdf

SEP. (2020b). Rechazo Cero. México: Secretaría de Educación Pública. Recuperado de: https:// rechazocero.sep.gob.mx/

Silva, M. y García, E. (21 de marzo 2020). Foros IBERO/El Derecho a la Educación Superior y la Equidad. México. Educación Futura. Recuperado de: http://www.educacionfutura.org/foros-ibero-elderecho-a-la-educacion-superior-y-la-equidad/

Torres, E. (15 de mayo 2020) Estudian el paso de la educación presencial a la educación en línea. iteso. Universidad Jesuita de Guadalajara. Recuperado de: https://iteso.org/web/general/ detalle?group_id=20312555

Vieyra, A., Belden, M., de la Calle, R. y Martínez, A. (2020). The impact of the covid-19 pandemic on higher education in Mexico, Colombia and Peru. EY-Parthenon. Recuperado de: https:// www.ey.com/en_gl/strategy/how-covid-19-has-impacted-higher-education-in-mexicocolombia-and-peru

Zabalgoitia, M. (2020). Géneros, equidad y violencias en tiempos de covid-19: ¿dónde quedan la educación y la universidad? En: Educación y pandemia. Una visión académica. Pp. 174-182. México: IISUE-UNAM. 